\title{
Successful Management of Right Ventricular Perforation Associated with a Pacemaker Lead During Off-Pump CABG Surgery: A Case Report
}

\author{
Masoud Tarbiat, ${ }^{1}$ Mohammad Hossein Bakhshaei, ${ }^{2}$ and Mehdi Moradi ${ }^{3,}{ }^{*}$ \\ ${ }^{1}$ Cardiac Aneasthesiologist, Fellowship of Cardiac Aneasthesia, Assistant Professor of Cardiac Aneasthesia, Clinical Research Development Unit of Farshchian Hospital, \\ Department of Anesthesiology, School of Medicine, Hamadan University of Medical Sciences, Hamadan, Iran \\ ${ }^{2}$ Cardiac Aneasthesiologist, Fellowship of Cardiac Aneasthesia, Associate Professor of Cardiac Aneasthesia, Clinical Research Development Unit of Farshchian Hospital, \\ Department of Anesthesiology, School of Medicine, Hamadan University of Medical Sciences, Hamadan, Iran \\ ${ }^{3}$ Cardiologist, Fellowship of Electro Physiology, Assistant Professor of Cardiology. Clinical Research Development Unit of Farshchian Hospital, Department of Cardiology, \\ School of Medicine, Hamadan University of Medical Sciences, Hamadan, Iran \\ "Corresponding author: Mehdi Moradi, Department of Cardiology, Farshchian Cardiovascular Surgery Center, Hamadan University of Medical Sciences, Shahid Fahmedeh \\ Street, Hamadan, Iran. Tel: +98-9122151560, E-mail: mmorad341@yahoo.com
}

Received 2017 March 10; Revised 2017 April 13; Accepted 2017 May 17.

\begin{abstract}
Introduction: Intraoperative right ventricular perforation due to pacing catheter after its successful and uneventful insertion is a rare complication. Here, we present a case of cardiac arrest due to right ventricular perforation associated with a pacemaker lead during off-pump coronary artery bypass graft surgery.

Case Presentation: The case was a 68-year-old male, who was admitted to our hospital with retrosternal chest pain. He had a history of implantation of a permanent pacemaker due to symptomatic complete atrioventricular block. Based on angiography, the diagnosis was 3- vessel disease involving the left anterior descending, second obtuse marginal, and right coronary arteries. The right ventricle was perforated by the tip of the permanent pacemaker lead during off-pump coronary artery bypass graft surgery. Subsequently, the patient suddenly experienced cardiac arrest and underwent emergency on-pump cardiac surgery.

Conclusions: This case showed that in some situations, emergency surgery as a life saving procedure may be required in cardiac perforation due to permanent pacemaker lead even following cardiac arrest.
\end{abstract}

Keywords: Pacemaker, Artificial, Coronary Artery Bypass, Off-Pump

\section{Introduction}

Right ventricular perforation by an endocardial lead is not common, but it is considered a potentially life threatening complication of pacemaker implantation (1). Because the right ventricular (RV) wall is thinner than the left, perforations to the RV is not surprising. Myocardial perforation may occur due to improper force on the leads against the RV free wall during implantation. "However, intraoperative RV perforation due to pacing catheter after its successful and uneventful insertion is a rare complication." (2). Here, we report a case of cardiac arrest due to RV perforation associated with a permanent pacemaker lead during off-pump coronary artery bypass graft(OPCAB) surgery that was treated successfully with emergency onpump coronary artery bypass graft (ONCAB) surgery.

\section{Case Presentation}

A 68-year-old male was admitted to the emergency room with retrosternal chest pain. A year ago, he underwent implantation of a dual chamber permanent pacemaker due to symptomatic complete atrioventricular block. Because he experienced pacemaker failure 2 months earlier, a single chamber permanent pacemaker was implanted in the right ventricle for him through right subclavian vein. Chest x-ray findings indicated a dual chamber leads through left subclavian vein and a single chamber permanent pacemaker lead through right subclavian vein in the right ventricle (Figure 1). The angiography report revealed that the patient had a patent left main artery (LMA) and a 3- vessel disease involving the left anterior descending (LAD), second obtuse marginal (OM), and right coronary arteries (RCA). Therefore, the patient was a candidate for elective OPCAB surgery after reprogramming the pacemaker to VOO mode.

Under general anesthesia with standard monitoring for cardiac anesthesia using a bipolar ESU (electro surgical unit) and after completion of the anastomosis LIMA to LAD while lifting the cardiac ventricular apex for anastomosis to OM artery, suddenly the RV was perforated by the tip of the ventricular lead of the previous dual chamber pacemaker (Figure 2). Then, blood pressure decreased rapidly and the patient unexpectedly experienced cardiac arrest. Immediately, the patient was resuscitated with administration of epinephrine (up to $4 \mathrm{mg}$ ), atropine (up to $2 \mathrm{mg}$ ), and internal cardiac massage for 7 minutes. After establish- 
ing $\mathrm{CPB}$ (cardio-pulmonary bypass), dexamethasone $8 \mathrm{mg}$, thiopental sodium $3 \mathrm{mg} / \mathrm{kg}$, lidocaine $1 \mathrm{mg} / \mathrm{kg}$, and magnesium sulfate $30 \mathrm{mg} / \mathrm{kg}$ were administrated intravenously according to our institutional protocol. When all the grafts anastomoses were performed successfully, the RV perforation was repaired with a pledgetted mattress suture, and the perforated lead was withdrawn slowly as the suture was tightened.

Subsequently, the patient was rewarmed and weaned off $C P B$ with the same single chamber permanent pacemaker (VOO Mode). The previous dual chamber pacemaker leads were not removed because of technical difficulties of extraction of the leads.

The operation was uneventful and the patient was extubated after 6 hours with the same permanent pacemaker (VOO Mode). He was completely alert with no hemodynamic complications. Finally, he was discharged from the hospital in a good overall condition, with the same single chamber permanent pacemaker (VVI mode).

\section{Discussion}

One of the potential complications of permanent cardiac pacing lead positioning is cardiac perforation, which can have severe clinical consequences including pericardial effusion, cardiac tamponade, pneumothorax, and death (3). RV perforation generally manifests during implantation or within 24 hours of implantation and has a prevalence rate of $0.1 \%$ to $6 \%(4,5)$. The predictors of lead perforation are temporary pacemaker implantation, corticosteroid use, active fixation leads, low body mass index, old age, female gender, and concomitant anticoagulation therapy $(6,7)$. In our case, the intraoperative predictors of lead perforation were old age, concomitant anticoagulation therapy, leads of 2 pacemakers, and excessive manipulation of heart during OPCAB. Perforation most often occurs in the apex or free wall of the RV outflow tract, frequently at the time of pressure by straight stylet during implantation, which is perpendicularly transmitted to the heart wall, and when the excessive length of the lead under tension is left and generates additional force leading to perforation. As expected during OPCAB, greater changes arise when the heart is positioned to expose the lateral wall compared with the anterior aspect of the heart $(6,7)$.

Patient management strategy should depend on the dynamics of symptoms, pericardial effusion, and hemodynamic status (3). Some clinicians propose that the diagnosis of lead perforation requires lead removal. However, some other clinicians suggest that the removal of a chronically perforated lead in the absence of pacemaker malfunction is not necessary (8). The implantation of epicardial leads should be considered in the case of open chest

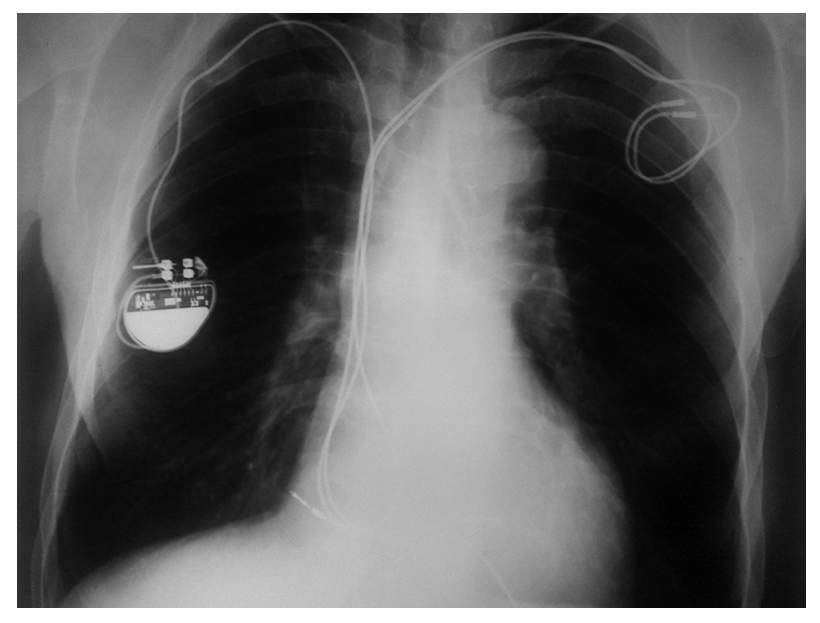

Figure 1. Pre-operative Chest $x$-Ray Showing a Dual Chamber Leads (Thick Arrow) and a Single Chamber Permanent Pacemaker (Narrow Arrow)

surgery. Chowdhry V et al. reported a case of intra operative $\mathrm{RV}$ perforation due to temporary pacing catheter during OPCAB surgery. Their patient did not suffer a cardiac arrest and managed successfully with epicardial pacing during OPCAB surgery (2). However, in our case, the patient suffered from cardiac arrest, and intraoperative RV perforation seemed to be related to having 2 pacemakers' leads and excessive manipulation of the heart during OP$\mathrm{CAB}$ surgery. Because cerebral injury is a major complication during cardiac arrest, thus a number of medications including steroids, magnesium sulfate, lidocaine and barbiturates, which seem to be useful in protecting the brain, were used (9).

\subsection{Conclusion}

Immediate changing from OPCAB to ONCAB surgery might be a life- saving method without any complication to adequately manage intraoperative right ventricular perforation accompanied by cardiac arrest even several minutes after cardiac arrest. Therefore, to avoid this complication, we advise an elective ONCAB surgery in such patients because it prevents the emergency conversion of OPCAB to ONCAB surgery, which has a very high mortality and morbidity. 


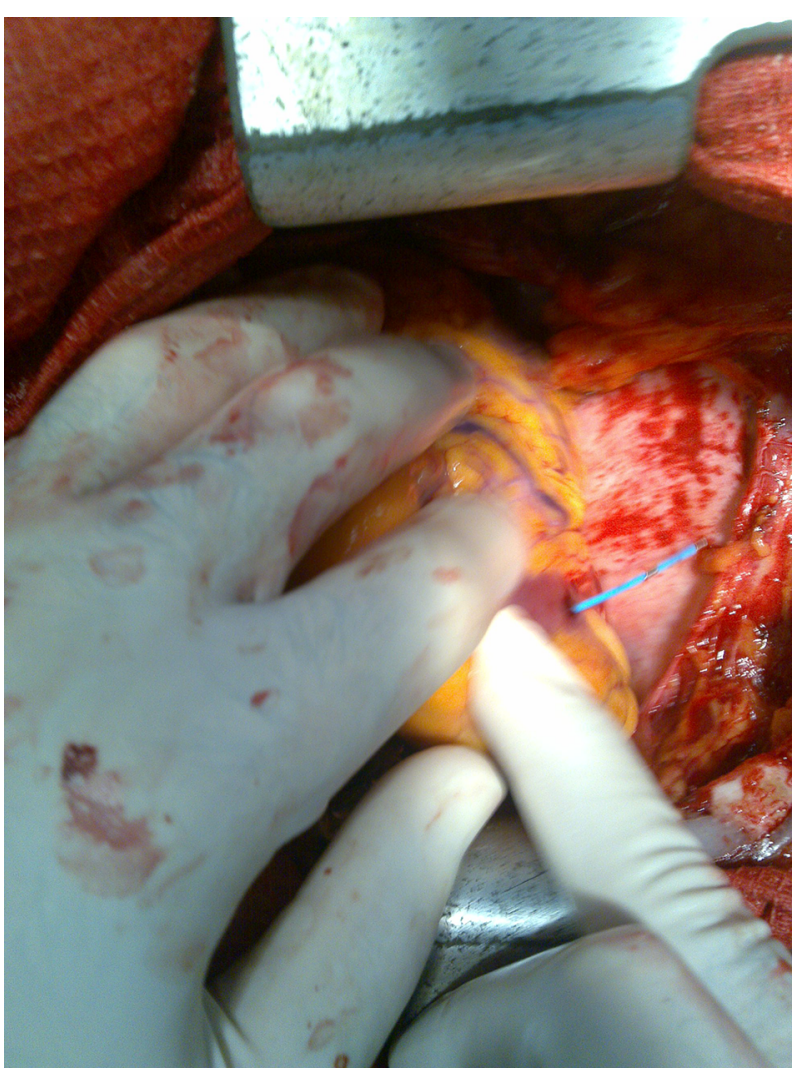

Figure 2. Intra-operative View of Right Ventricular Perforation Associated With a Pacemaker Lead

\section{Acknowledgments}

The authors thank Dr. Mohammad Qomeyssi and anesthesiology nurses, who contributed to the management of the patient in the operating room.

\section{Footnotes}

Authors' Contribution: Masoud Tarbiat managed the patient in the operation room; Mohammad Hossein Bakhshaei and Masoud Tarbiat prepared the manuscript; Mehdi Moradi, study supervision.

Conflicts of Interest: The authors have no conflicts of interest.

Financial Disclosure: There was no conflict of interest.

Funding/Support: This study did not receive any fund or financial support.

\section{References}

1. Yavari A, Khawaja ZO, Krishnamoorthy S, McWilliams ET. Perforation of right ventricular free wall by pacemaker lead detected by multidetector computed tomography. Europace. 2009;11(2):252-54. doi: 10.1093/europace/eun381.

2. Chowdhry V, Biswal S. Transvenous pacing and manipulation of heart in coronary artery bypass grafting: A word of caution. Indian J Anaesth. 2013;2(57):204-6.

3. Welch AR, Yadav P, Lingle E, Lintonfrazier L, Haouzi A, Wolbrette E. Sub acute right ventricular pacemaker lead perforation: Often talked about in consent forms but very rarely seen. J Innovations Cardiac Rhythm Manag. 2011;2:442-5.

4. Oda T, Kono T, Akaiwa K, Takahara Y, Yasuoka C, Nakamura K. Surgical repair of subacute right ventricular perforation after pacemaker implantation. Case Rep Cardiol. 2017;2017:3242891. doi: 10.1155/2017/3242891.

5. Akbarzadeh MA, Mollazadeh R, Sefidbakht S, Shahrzad S, Bahrololoumi Bafruee $\mathrm{N}$. Identification and management of right ventricular perforation using pacemaker and cardioverter-defibrillator leads: A case series and mini review. J Arrhythm. 2017;33(1):1-5. doi: 10.1016/j.joa.2016.05.005. [PubMed: 28217220].

6. Maziarz A, Zabek A, Malecka B, Kutarski A, Lelakowski J. Cardiac chambers perforation by pacemaker and cardioverter-defibrillator leads. Own experience in diagnosis, treatment and preventive methods. Kardiol Pol. 2012;70(5):508-10. [PubMed: 22623248].

7. Hemmerling TM, Romano G, Terrasini N, Noiseux N. Aneasthesia for off-pump coronary artery bypass surgery. Annals Cardiac Aneasthesia. 2013;16(1):28-39.

8. Banaszewski M, Stepinska J. Right heart perforation by pacemaker leads. Arch Med Sci. 2012;8(1):11-3. doi: 10.5114/aoms.2012.27273. [PubMed: 22457667].

9. Tarbiat M, Sedighinejad A, Manafi B, Farhanchi A, Imantalab V, Mirmansouri A. Deep hypothermic circulatory arrest (DHCA) in a man undergoing aortic aneurysm dissection surgery. Iran Heart $J$. 2012;13(3):46-53. 